\title{
A novel single-photon detector based on capacitance-balancing technique
}

\author{
Xie Chao ${ }^{1,}$,, Yanli Zhao ${ }^{1}$ \\ ${ }^{1}$ Wuhan National Laboratory of optoelectronics, Huazhong University of Science and Technology, \\ Wuhan 430074, China \\ aM201372515@hust.edu.cn
}

Keywords: InGaAs/InP APD, capacitance balancing, single photon detector.

\begin{abstract}
A gated InGaAs/InP single-photon detector based on a novel capacitance balancing technique was demonstrated. The single-photon detector is based on a gated InGaAs/InP avalanche photodioe. A quantum efficiency of $10 \%$ at $1550 \mathrm{~nm}$ was obtained with a dark count probability per gate of $1.82 \times 10^{-6}$ and an afterpulsing probability of $3.6 \%$ at a detection rate of $100 \mathrm{MHz}$. Moreover, compared with traditional capacitance balancing technique, our scheme can reduce dark count probability obviously.
\end{abstract}

\section{Introduction}

Near infrared single-photon detectors (SPDs) are widely used in many fields, such as quantum secure communication, astronomy, and ultrasensitive spectroscopy. The InGaAs/InP avalanche photodiode (APD) has been the most practical device for SPDs at telecommunication wavelength[1]. Since a photo-excited carrier grows into a macroscopic current output via the carrier avalanche multiplication in an APD operated in the Geiger mode, a single photon can be detected efficiently. However, some carriers trapped in the APD are subsequently emitted, and trigger additional avalanches that cause erroneous events. The InGaAs/InP APD in Geiger mode has a particular high probability that these so called "afterpulses" occur. Therefore, the InGaAs/InP APD is usually operated in the gated mode in which the gate duration (gate-on time) is generally set to a few nanoseconds. Then the interval between two consecutive gates is set to more than the lifetime (in orders of microseconds) of the trapped carriers so that the afterpulse is suppressed.

The alternative SPDs at telecommunication wavelengths are frequency-upconversion-assisted Si-APD (upconversion detector) and a superconducting single-photon detectors (SSPD). Although these SPDs can be operated with greater voltages than gigahertz clock systems, they have drawbacks that make them difficult to apply to practical QKD systems. The upconversion detectors suffers from background noise counts with high detection efficiency, while the SSPD requires cryogenic below $4 \mathrm{~K}$.

Thus far,several techniques, such as self-differencing technique[2], and sinusoidal gating technique[3], have been invented for InGaAs/InP single-photon avalanche photodioses(SPADs) in the gated Geiger mode, increasing the working speed over GHz. With these methods, SPADs can sense much weaker photon-induced avalanches at a high speed with good performance. However, it is quite difficult to change the gating frequencies of these SPADs, limiting their applications. Here, we propose the capacitance-balancing technique to solve the problem, easily tuning the gating freque- ncies without changing any state of the SPAD. At a detection rate of $200 \mathrm{MHz}$, we obtained a single photon detection efficiency of $10 \%$ with an afterpulse probability of $3.6 \%$ and a low dark count rate $\left(1.82 \times 10^{-6}\right.$ per pulse). These make the device suitable for quantum secure communication, which requires that the bit error rate is less than $5 \%$. 


\section{Experimental setup}

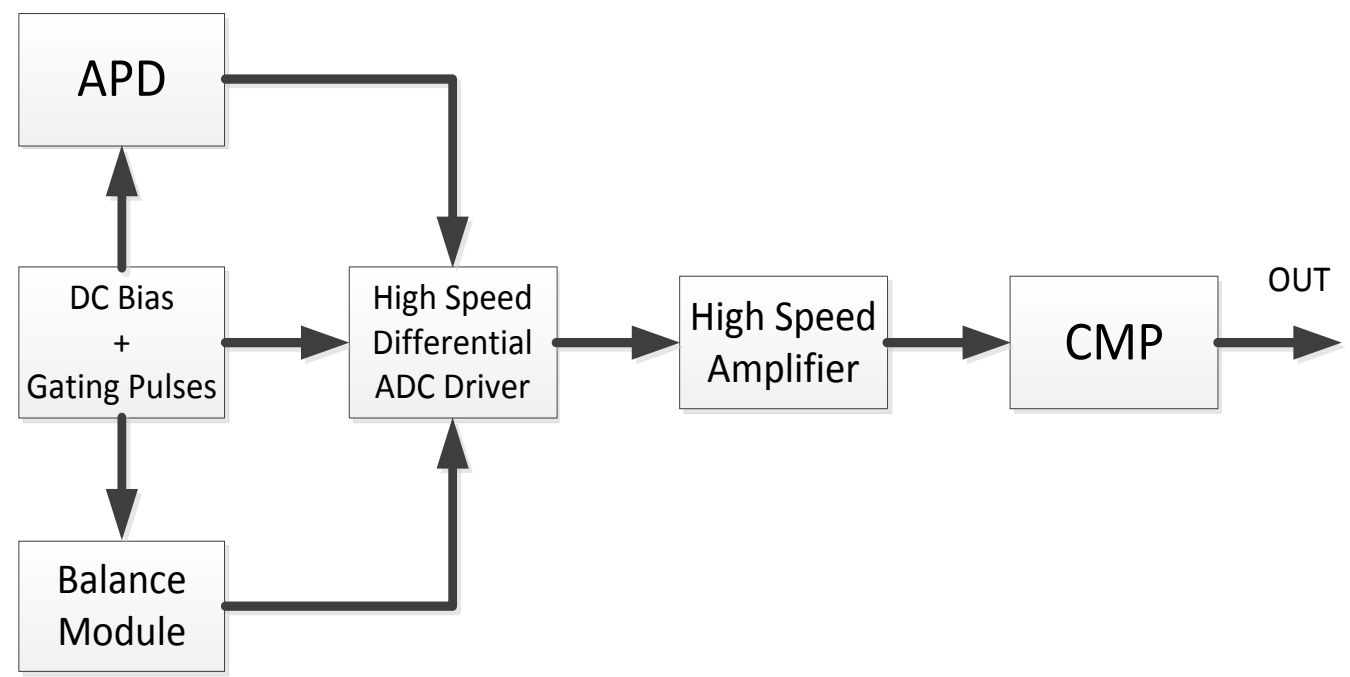

Figure.1.Schematic of the capacitance-balancing single photon detector.

A diagram of our SPD is shown in Figure 1.The tested APD is PGA300 (Princeton Lightwave). The APD is cooled by a three-stage peltier. The APD module was used as the single-photon detection element after it was fiber pigtailed. In order to supply an AC voltage superposed on the DC reverse bias voltage $V_{D C}$ to the APD, we used a gated passive quenching circuit (GPQC). The sinusoidal voltage at a frequency of $w g$ was produced by a signal generator (SG:Agilent 83630B) and used as the gated voltage. Here the balance module was used to produce the similar response as the APD. The avalanche signal was discriminated by the ultra high speed comparator (CMP:MAXIM MAX9600). We finally obtained the logic output in the emitted-coupled-logic (ECL) level.

We evaluated the SPD performance for weak laser pulses at $1550 \mathrm{~nm}$. The laser pulses were triggered by the clock signal given by the SG. Here the phase of the sinusoidal gate voltage was synchronized with that of the $10 \mathrm{MHz}$ standard clock. The relative phase delay between the sinusoidal voltage and the laser pulse was adjusted by the phase shifter. In order to obtain the highest quantum efficiency, the timing ( laser pulse delay ) of the optical pulses at the APD must be carefully tuned by the phase shifter.

To evaluate the quantum efficiency $\eta$, the dark count probability $P_{d}$, and the afterpulsing probability $P_{a}$ of our SPD , the detection events (logic outputs from the SPD) were registered by a multi-channel scaler that gave the time histogram of the detection events. As described above, the the repetition frequency $w_{L}$ of the laser pulses was set to $10 \mathrm{MHz}$, which was lower than the frequency $w g$ of the sinusoidal gate. Therefore, there are many nonilluminated gates , and the photon detection events occur only in the illuminated gates, as shown in Fig.2. The number of events in the illuminated gates is consequently much larger than that in the nonilluminated gates (see Histogram in Fig.2), since only dark counts and afterpulse events occur in the nonilluminated gates. The histogram of the dark counts can be easily obtained with the laser switched off (see Histogram in Fig.2). We must note that there are actually not only the dark counts but also the afterpulse events correlated with the dark count events. However, such afterpulse events were treated here as dark count events. The number of events in nonilluminated gates with the laser switched on is higher than that with the laser switched off, since afterpulse events correlated with the photon detection events occur. The afterpulsing probability $P_{a}$, defined as the ratio of the total afterpulse events to photon detection events, is given by the expression.

$$
P a=\frac{\left(C_{N I}-C_{D}\right) R}{C_{I}-C_{N I}},
$$




$$
R=\frac{\mathrm{w}_{\mathrm{g}}}{\mathrm{w}_{\mathrm{L}}},
$$

Where $C_{N I}$ and $C I$ are the number of the events per nonilluminated gate, respectively, and $C D$ is the number of events per gate with the laser switched off. $R$ is the ratio of the gating frequency of the laser pulse repetition frequency, and corresponds to the number of the gates that are applied to the APD during one period of the laser pulse.

The quantum efficiency and the dark count probability are easily evaluated using the relations

$$
\begin{gathered}
\mu \eta N \mathrm{~s}=\mathrm{C}_{\mathrm{I}}-\mathrm{C}_{\mathrm{NI}}, \\
P \mathrm{~d} N \mathrm{~s}=C_{D}
\end{gathered}
$$

Where $\mu$ is the average photon number per optical pulse, and $N s$ is the number of the measurements using the MCS. In our experiment, $\mu$ and $N s$ was set to 0.1 and $10^{8}$, respectively.

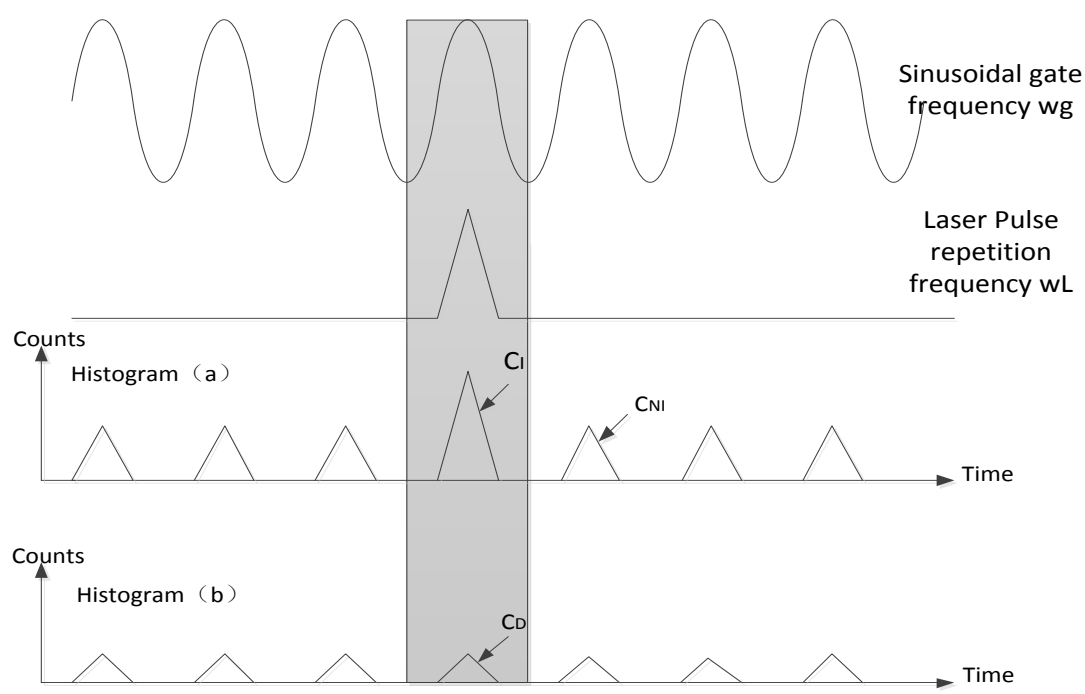

Fig.2.Schematic diagram of the histogram measurements. The illuminated gates are highlighted. Histogram (a) and (b) show the time histograms of the detection events when the laser was switched on and off, respectively. $C_{I}$ and $C_{N I}$ denote the number of events in the illuminated and nonilluminated gates, respectively, with the laser switched on. $C_{D}$ denotes the number of events per gate with the laser switched off.

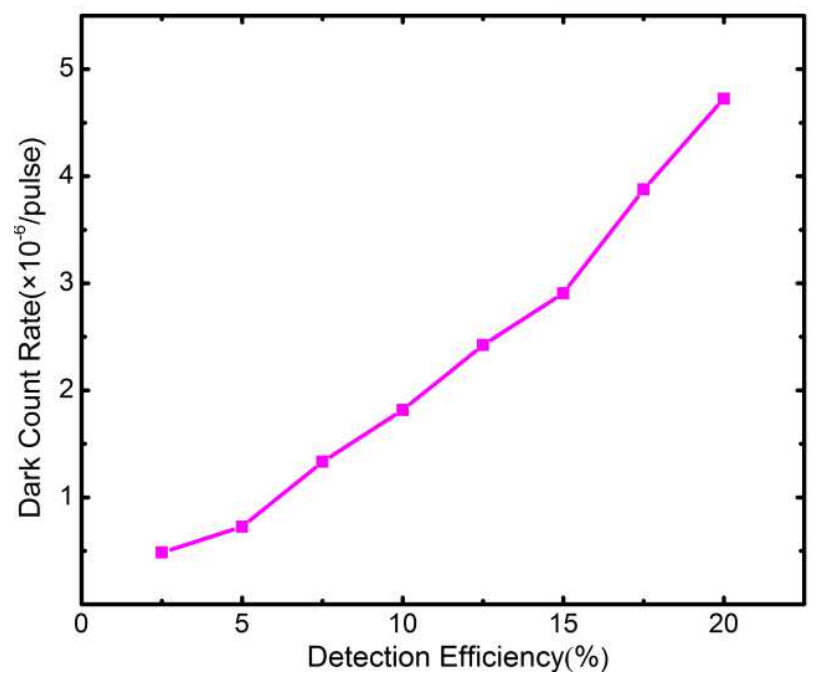

Fig.3.Dark count rate versus detection efficiency

Figure 3 shows the relation between dark count rate and the detection efficiency. It can be seen that the dark count rate rise smoothly when the detection efficiency is relatively low. The dark count rate 
was $7.26 \times 10^{-7}$ per gate when the detection efficiency was $5 \%$, then it rose to $2.9 \times 10^{-6}$ per gate when the detection efficiency was $15 \%$. Nevertheless, when the DC bias increase to a high degree, the dark count rate rise sharply. The dark count rate at $20 \%$ detection efficiency was $4.72 \times 10^{-6}$ per gate.

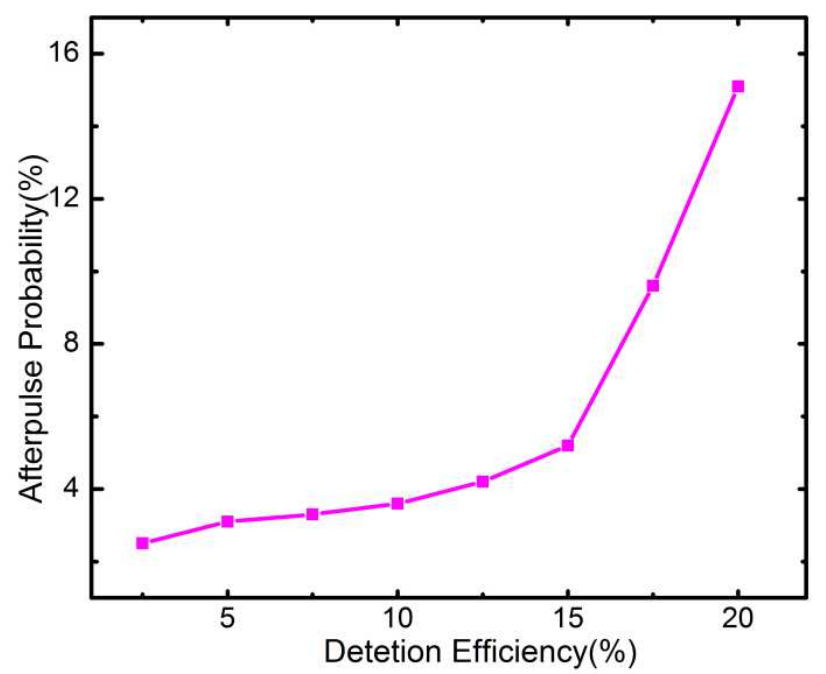

Fig.4. Afterpulse probability versus detection efficiency

Figure 4 shows the relation between afterpulse probability and the detection efficiency. We can also see that the afterpulse probability rise slowly when the detection efficiency is relatively low. The afterpulse probability at $5 \%$ detection efficiency was approximately $3.1 \%$, and it rose to $5.2 \%$ when the detection efficiency was $15 \%$. However, when the detection efficiency exceeded $15 \%$, the afterpulse probability rise sharply. The afterpulse probability at 20\% detection efficiency was $15.1 \%$.

\section{Summary}

In conclusion, we have developed a single-photon detector at 1550nm using a sinusoidal gated capacitance balancing InGaAs/InP APD. A gated repetition of $100 \mathrm{MHz}$ was achieved with high quantum efficiency, low dark count probability, and low afterpulsing probability. The single-photon detector can be easily applied to QKD systems.

\section{Acknowledgements}

This work was supported by the National Hi-Tech Research and Development Program of China (Grand No. 2008AA01Z207), Natural Science Foundation of Hubei Province, China (Grant No. 2010CDB01606), Fundamental Research Funds for the Central Universities (2014KXYQ013), Huawei Innovation Research Program (YJCB2010032NW, YB2012120133, YB2014010026) and Scientific Research Foundation for the Returned Overseas Chinese Scholars.

\section{References}

[1]Marsili, F., Verma, V. B., Stern, J. A., et al., Detecting Single Infrared Photons with 93\% System Efficiency, Nature Photonics 7, 210-214 (2013).

[2]H. Takesue, E. Diamanti, C. Langrock, M. M. Fejer, and Y. Yamamoto, $1.5 \mu \mathrm{m}$ photon-counting optical timedomain reflectometry with a single-photon detector based on upconversion in a periodically poled lithium niobate waveguide, Opt. Lett. 31, 727-729 (2006)

[3]N. Namekata, G. Fujii, T. Honjo, H. Takesue, and S. Inoue, Quantum key distribution using single-photon detectors based on a sinusoidally gated InGaAs/InP avalanche photodiode, Appl. Phys. Lett. 91, 011112 (2007) 